\title{
Comparison of Abnormal Cholesterol in Children, Adolescent \& Adults in the United States, 2011-2014: Review
}

\author{
Rasaki Aranmolate1* \\ 1 School of Public Health, Jackson State University, MS, USA \\ *Corresponding Author: ayodeji88@yahoo.co.uk \\ Citation: Aranmolate, R. (2017). Comparison of Abnormal Cholesterol in Children, Adolescent \& Adults \\ in the United States, 2011-2014: Review. European Journal of Environment and Public Health, 1(1), 04. \\ https://doi.org/10.20897/ejeph.201704
}

Published: July 21, 2017

\begin{abstract}
The purpose of this paper is to compare the prevalence of abnormal cholesterol in children, adolescent and adults in the United States. Data from the National Health and Nutrition Examination survey (NHANES) for 2011-2014 were obtained for the comparison. A t test statistical analysis was conducted at a significant level of $\mathrm{p}<0.05$ to check for differences in the groups. The results of high total cholesterol and low high density lipoprotein (HDL) were compared in children, adolescent and adults according to race, sex and ethnicity. A total of $27.9 \%$ of men had low level of HDL cholesterol, $10 \%$ of women had low HDL, $13.4 \%$ of children and adolescent had low level of HDL. Boys had low HDL (14.8\%) while girls had $12 \%$. The non-Hispanic black adults with elevated total high cholesterol was $8.6 \%$, non-Hispanic white $(12.5 \%)$, Hispanic adults $(13.1 \%)$ while non-Hispanic black children and adolescent had elevated total high cholesterol, the non-Hispanic white and Hispanic adolescent and children had low total high cholesterol. The level of low HDL cholesterol was higher in children, adolescent and adult male population, the high total cholesterol was higher in the female children, adolescent and adults.
\end{abstract}

Keywords: total cholesterol, HDL cholesterol, cardiovascular disease, NHANES

Abbreviations: CVD: Cardiovascular disease; NHLBI: National Heart, Lung and Blood Institute; HDL: High density lipoprotein; NHANES: National Health and Nutrition Examination Survey; CDC: Centers for Disease Control and Prevention; U.S: United States; NCHS: National Center for Health Statistics.

\section{INTRODUCTION}

Cardiovascular disease remains the highest cause of death worldwide with more than 17 million deaths recorded annually (WHO, 2014). It occurs more often in the elderly individual but it is less likely to occur before 60 years of age (Berenson, 1998). However, the cause of high cholesterol leading to cardiovascular disease (CVD) usually starts at an early year of life specifically during childhood and adolescent (Berenson, 1998). The risk factors for CVD and risky behaviors can be identified during childhood and adolescent age and the level of these factors have been known to be associated with the consequences of atherosclerosis in children and adult (Berenson, 1998). Some studies revealed the risk factors associated with CVD and certain behaviors during childhood, which can later manifest as a serious CVD in adult life (Chen, 2008). In addition, elevated amount of high total cholesterol and low level of blood high-density lipoprotein (HDL) are risk factors for developing coronary heart disease (Assmann, 1996). Some researchers made a case that CVD can be prevented early, if physicians can make early identification of risk factors and behaviors (McGill, 2008). Furthermore, they observed that CVD can begin at an early age and made a recommendation for early intervention by the clinicians (McGill, 2008). The United States National Heart, Lung and Blood Institute made a recommendation recently that CVD risk factors should be 
screened universally and should be started during childhood (NHLBI, 2013). In addition, the cardiovascular health of the United States can be improved if there is a standard clinical guide on recommended screening for children and adolescent for risk factors associated with CVD, specifically for abnormal cholesterol levels in the blood (McGill and McMahan, 2008).

In this review, the comparison of abnormal cholesterol in children, adolescent and adults will focus on the assessment of prevalence of abnormal high total cholesterol and low HDL cholesterol during four-year period (2011-2014) (Nguyen and Carroll, 2015).

\section{METHOD}

The data used for this analysis were obtained from the National Health and Nutrition Examination Survey (NHANES) from 2011-2014 (Nguyen, Kit and Carroll, 2015). The studies were conducted by Centers for Disease Control and Prevention's (CDC) National Center for Health Statistics (NCHS) (Nguyen et al., 2015). A crosssectional survey was used to determine the health and nutritional status of the noninstitutionalized civilian population living in the United States (Nguyen et al., 2015). A complex, multiphase probability sampling method was used for these studies (Nguyen et al., 2015). The content of the survey had interview section that were conducted in the homes of the participants (Nguyen et al., 2015). In addition, it consists of detailed physical examination, including collection of standardized laboratory samples conducted in mobile examination centers (Carroll, Fryar and Kit, 2015). The survey was conducted in 2011-2014 (4-year period) which focuses on the nonHispanic black, non-Hispanic Asian, and Hispanic individuals and other groups (Carroll et al., 2015). The population were oversampled to achieve a dependable outcome for the subgroups (Carroll et al., 2015). The data were analyzed using SAS 9.3 with SUDAAN 10.0 which measured standard errors using Taylor Series Linearization to obtain the complex sample design (Nguyen et al., 2015). Furthermore, the examination sample weights, which is responsible for selecting and modifying nonresponse and uncovered, were used in calculating the prevalence of elevated high total cholesterol and low HDL cholesterol (Nguyen et al., 2015). The study only measured the level of cholesterol and it did not check participants on cholesterol lowering medications (Nguyen et al., 2015). A t test statistical analysis was used at a significant level of $\mathrm{p}<0.05$ to check for variation in the groups (Nguyen et al., 2015).

\section{DEFINITIONS}

High total cholesterol (adult): Serum total cholesterol equal or greater than $145 \mathrm{mg} / \mathrm{dl}$ (Carroll et al., 2015). High total cholesterol (children \& Adolescent): Serum total cholesterol equal or greater than $240 \mathrm{mg} / \mathrm{dl}$ (Nguyen et al., 2015).

Low HDL cholesterol (adult): Serum total cholesterol equal or greater than $145 \mathrm{mg} / \mathrm{dl}$ (Carroll et al., 2015).

Low HDL cholesterol (children \& Adolescent): Serum total cholesterol equal or greater than $145 \mathrm{mg} / \mathrm{dl}$ (Nguyen et al., 2015).

Healthy People 2020; Stroke and Heart Disease, Object 7: Reduction in the number of adults with high total serum cholesterol levels targeted at 13.5\% (Klein, 2001).

\section{RESULTS}

\section{Percentage of children, adolescent and adults with high total cholesterol between 2011-2014}

The prevalence of high total cholesterol among children and adolescent living in America was 7.4\% (Figure 1) (Nguyen et al., 2015) while the prevalence was 12.0\% in adults (Figure 2) (Carroll et al., 2015). In addition, adolescent aged 16 and 19 had frequency of $8.9 \%$ in high total cholesterol while children aged 6-8 was $6.0 \%$ (Figure 1) (Nguyen et al., 2015). There was a significant difference for children and adolescent aged 16-19, normal and overweight at $\mathrm{p}<0.05$ (Figure 1) (Nguyen et al., 2015). In the adult population, the frequency of high total cholesterol between ages of 20 and 39 was $7.5 \%, 40-59$ had $16 \%$ while 60 and above was $12.9 \%$ (Figure 2) (Carroll et al., 2015). Furthermore, men aged 40-59 had high total cholesterol of 15.3\% which is higher than aged 20-39 $(8.2 \%)$ and $7.3 \%$ for 60 and above (Carroll et al., 2015). The prevalence of high total cholesterol based on gender was low in boys at $5.9 \%$ and statistically significant at $\mathrm{p}<0.05$ than in girls (8.9\%) (Figure 1) (Nguyen et al., 2015). The prevalence of high total cholesterol was higher in children and adolescent who are obese (11.6\%) as compared to normal weight $(6.3 \%)$ and overweight $(6.9 \%)$ (Figure 1) (Nguyen et al., 2015). The percentage of high total cholesterol was lower in men (10.6\%) than women (13\%) (Figure 2) (Carroll et al., 2015). The percentage of participants in the age group of 20-39 and 40-59 shows no difference by sex but a lower percentage was recorded 


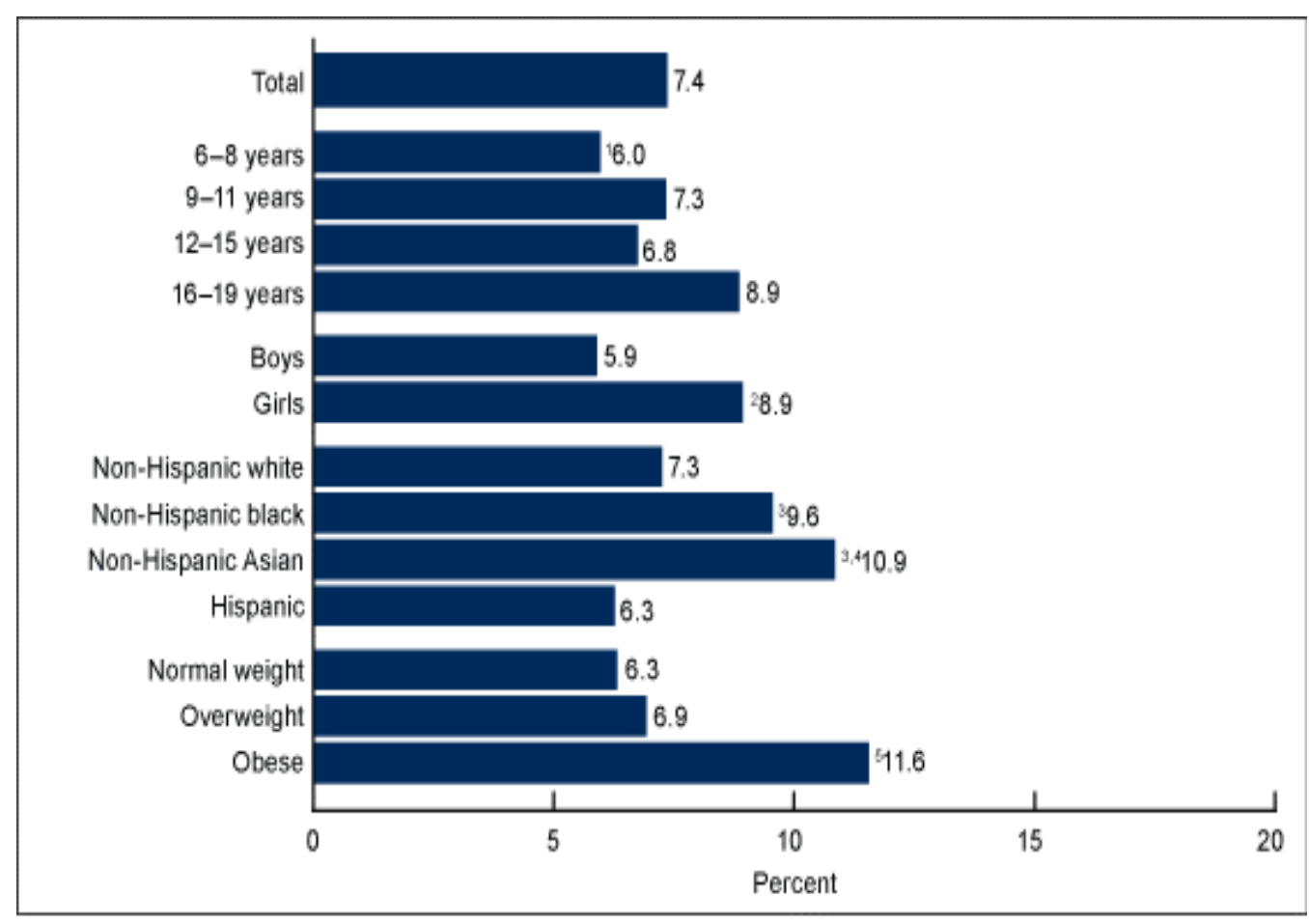

Figure 1. Frequency distribution of high total cholesterol among children and adolescents between 6-19 years by age, gender, race, Hispanic origin, and body mass index.

NOTES: Body mass index percentiles were grouped as at or greater than 5th up to 85th (normal weight), at or more than 85th up to 95th (overweight), and at or greater than 95th (obese) (Nguyen et al., 2015). SOURCE: CDC/NCHS, National Health and Nutrition Examination Survey, 2011-2014.

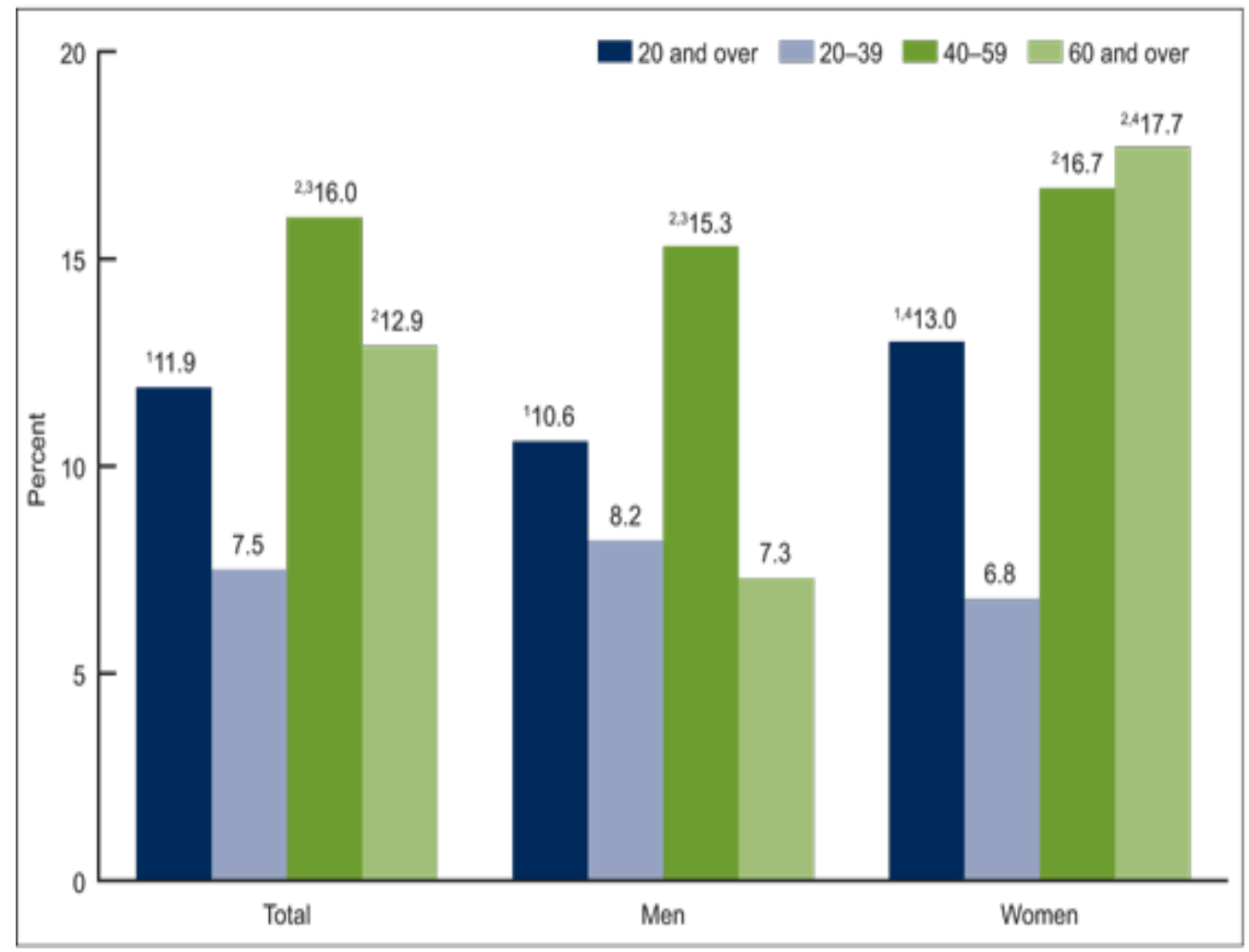

Figure 2. Frequency distribution of high total cholesterol in adults aged 20 and over, by age and gender. SOURCE: CDC/NCHS, National Health and Nutrition Examination Survey, 2011-2014.

for men who were 60 and above (7.3\%) than women in the same age group (17.7\%) (Figure 2) (Carroll et al., 2015). The crude estimate for men was $10.7 \%, 13.5 \%$ for women and the total was $12.1 \%$. There was a significant difference among the age group 20-39 and 60 years and above (Carroll et al., 2015). 


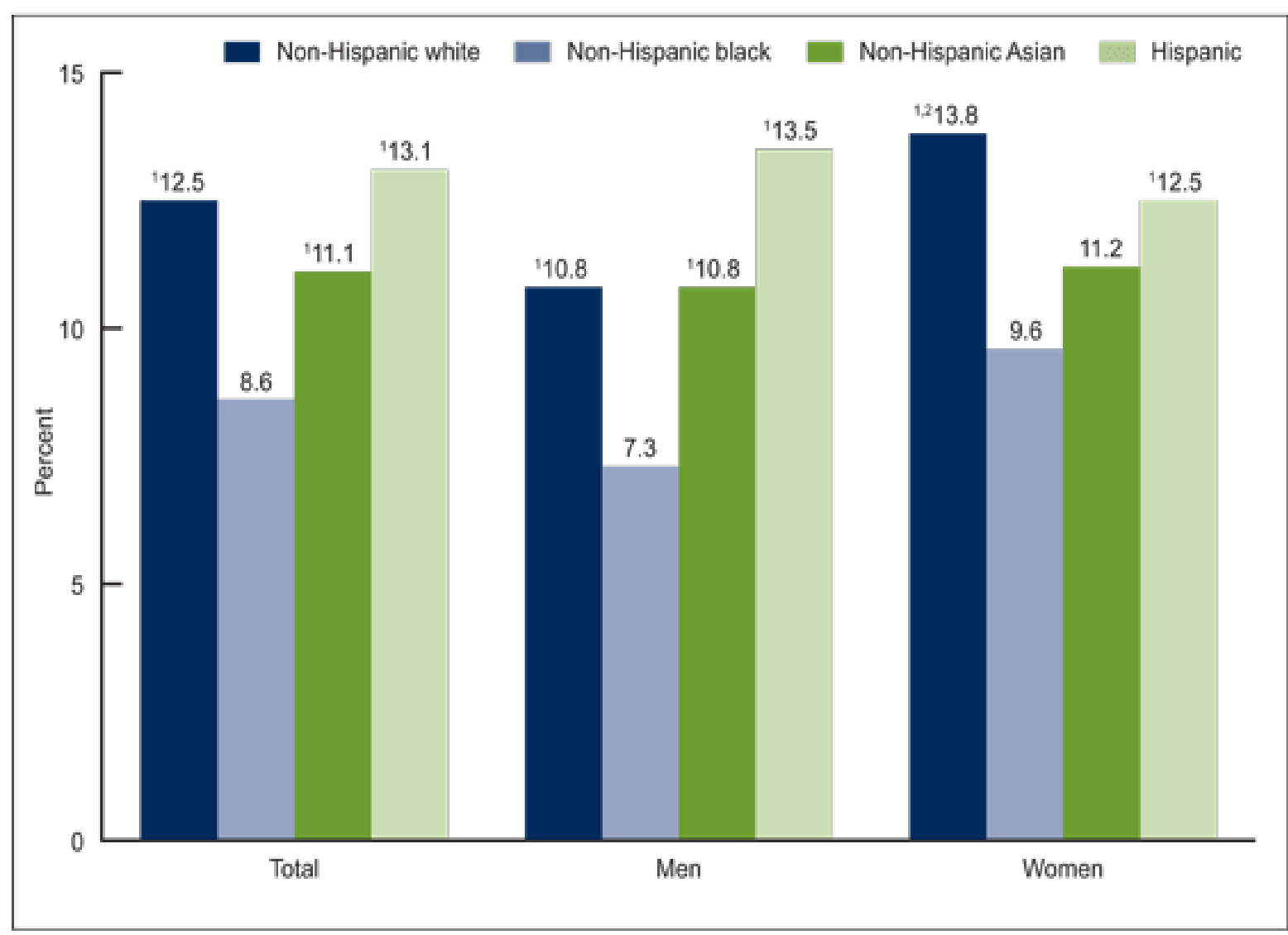

Figure 3. Prevalence of high total cholesterol among adults aged 20 and over, by gender, race and Hispanic origin adjusted by age.

SOURCE: CDC/NCHS, National Health and Nutrition Examination Survey, 2011-2014.

\section{Percentage of children, adolescent and adults with high total cholesterol by gender, race and Hispanic origin between 2011-2014}

The prevalence of high total cholesterol was $9.6 \%$ in non-Hispanic black, $10.9 \%$ in non-Hispanic Asian children which is higher when compared to non-Hispanic white with 7.3\% and $6.3 \%$ in Hispanic children (Figure 1) (Nguyen et al., 2015). In the adult population, non-Hispanic black had a high total cholesterol of $8.6 \%$ which is lower than non-Hispanic white (12.5\%) (Figure 3) (Carroll et al., 2015). Furthermore, non-Hispanic Asian was $11.1 \%$ which is lower than the Hispanic adults (13.1\%) (Figure 3) (Carroll et al., 2015). In addition, non-Hispanic white and Hispanic children had the lowest total high cholesterol, non-Hispanic black and non-Hispanic Asian adult had the lowest high total cholesterol (Carroll et al., 2015).

\section{Percentage of children, adolescent and adults with low HDL cholesterol by race, gender and Hispanic origin between 2011-2014}

The non-Hispanic white children and adolescent had 14.4\% of low HDL cholesterol which is higher than the non-Hispanic black with 7.4\% and non-Hispanic Asian (8.2\%) while the Hispanic had 15.6\% which is higher than the previous (Figure 5) (Nguyen et al., 2015). The prevalence of low HDL cholesterol was low in non-Hispanic black (13.6\%) and non-Hispanic Asian (15.2\%) adults while 19.1\% was observed in non-Hispanic white and 21.1\% in the Hispanic adults (Figure 4) (Carroll et al., 2015). Furthermore, non-Hispanic black men had a low prevalence of low HDL (20.7\%), non-Hispanic Asian was 25\% and Hispanic men had 30.7\% (Figure 4) (Carroll et al., 2015). Moreover, low HDL prevalence was lower in non-Hispanic black (8\%) and non-Hispanic Asian women (6.7\%) when compared with the non-Hispanic white (10.3\%) and Hispanic women (11.8\%) respectively (Carroll et al., 2015). The women had low percentage of low HDL cholesterol than men of the same race and ethnic group (Figure 4) (Carroll et al., 2015). The Hispanic children, adolescent and adults had elevated low HDL cholesterol than other ethnic group. The results of the analysis revealed a significant difference for both children and adult Hispanic and non-Hispanic white at $\mathrm{p}<0.05$ (Nguyen et al., 2015). Furthermore, there was a significant difference in the non-Hispanic Asian, non-Hispanic black and Hispanic men (Figure 4) (Carroll et al., 2015). 


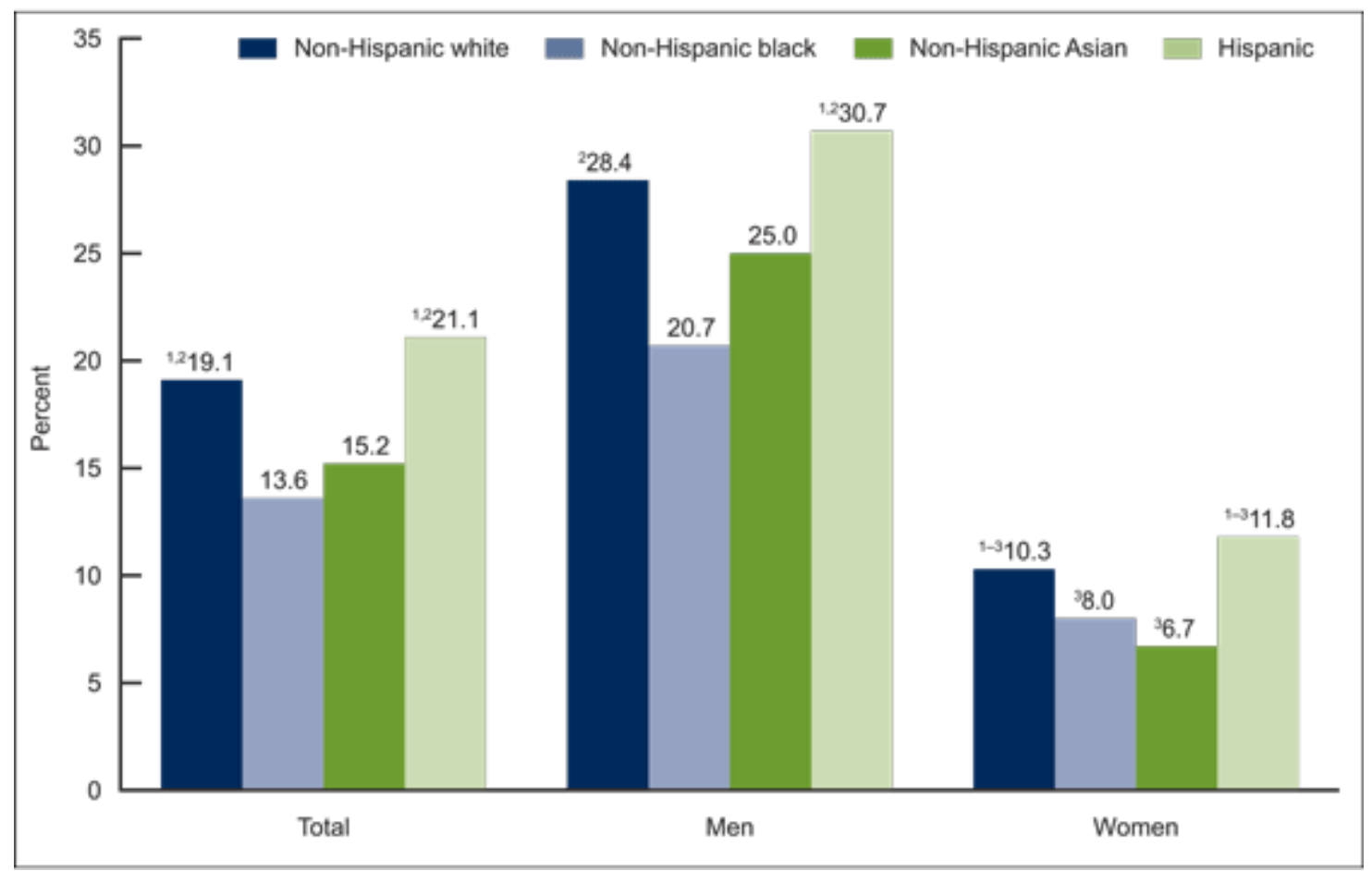

Figure 4. Prevalence of low HDL cholesterol among adults aged 20 and over, by gender and race and Hispanic origin adjusted by age.

SOURCE: CDC/NCHS, National Health and Nutrition Examination Survey, 2011-2014.

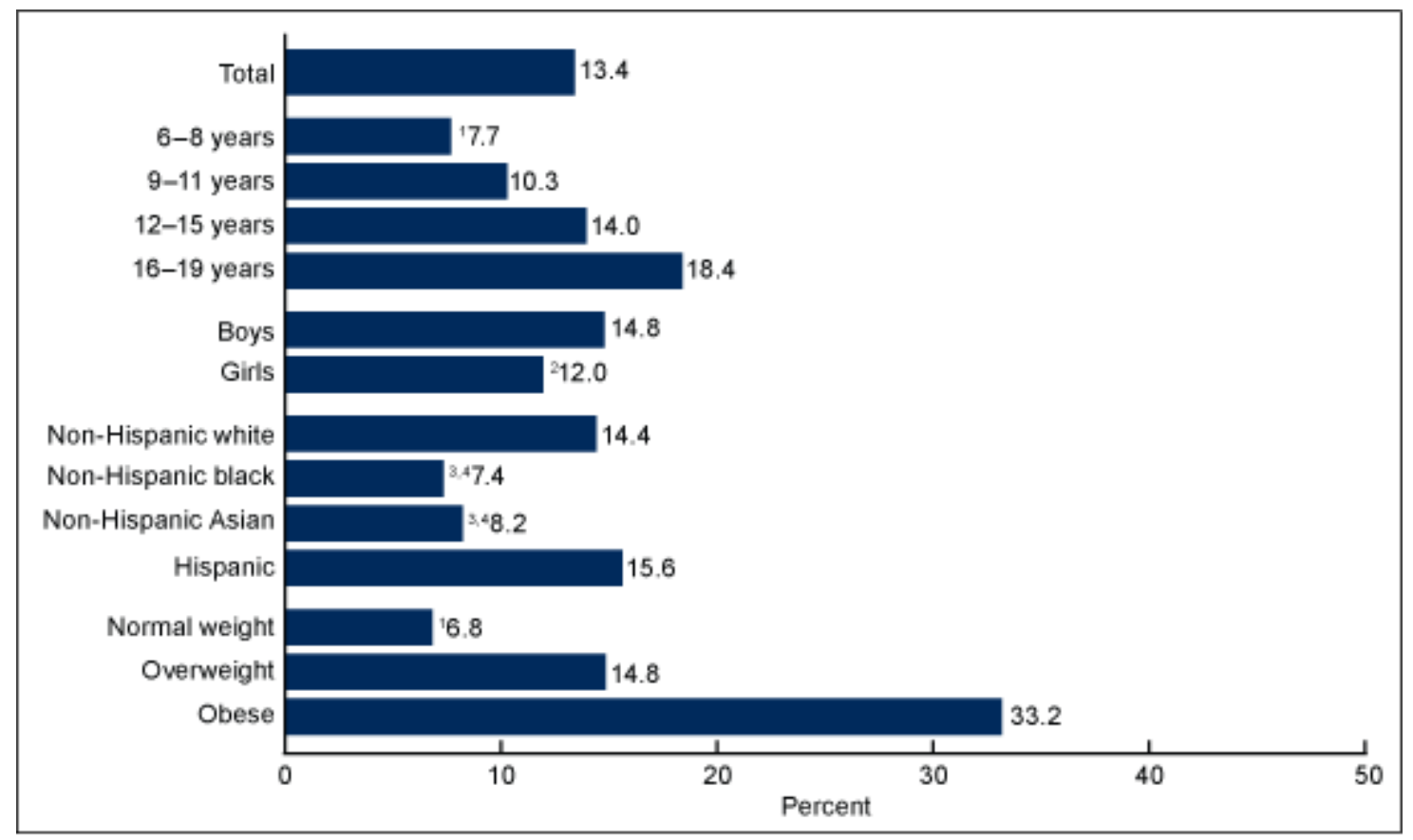

Figure 5. Frequency distribution of low HDL cholesterol among children and adolescent aged 6-19 years by age, gender, race and Hispanic origin, and body mass index.

NOTES: Body mass index percentiles were grouped as at or greater than 5th up to 85th (normal weight), at or more than 85th up to 95th (overweight), and at or greater than 95th (obese) (Nguyen et al., 2015). SOURCE: CDC/NCHS, National Health and Nutrition Examination Survey, 2011-2014.

\section{Percentage of children, adolescent and adults with low HDL cholesterol between 2011-2014}

A total of 13.4\% children and adolescent had low HDL cholesterol (Figure 5) (Nguyen et al., 2015). A rising trend for low HDL was observed in children aged 6-8 (7.7\%) and the adolescents aged 16-19 was 18.4\% (Figure 5) (Nguyen et al., 2015). The boys had low HDL of $14.8 \%$ which is higher than the girls (12\%) (Figure 5) (Nguyen et al., 2015). In addition, adults that were 18 years and above had 18\% of low HDL during the 4-year period. 


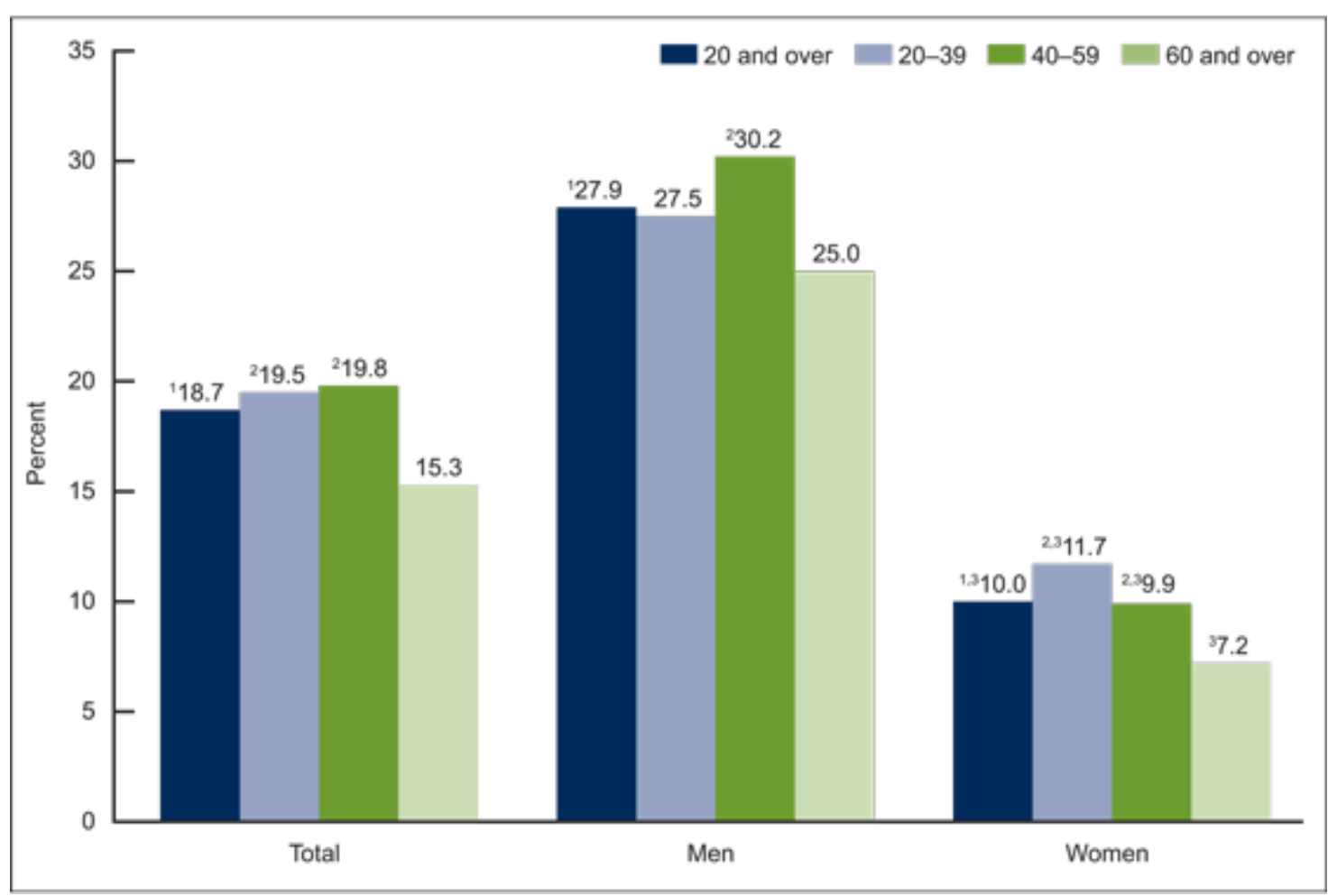

Figure 6. Frequency distribution of low HDL cholesterol among adults aged 20 and over, by gender and age in the United States.

SOURCE: CDC/NCHS, National Health and Nutrition Examination Survey, 2011-2014.

Children with normal weight had low HDL of $6.8 \%$, overweight had $14.8 \%$ while obese children had $33.2 \%$ of low HDL (Figure 5) (Nguyen et al., 2015). Participants aged 60 and above had 15.3\% of low HDL cholesterol which is lower than 19.5\% in aged 20-39 and 40-59 (19.8\%) (Figure 6) (Carroll et al., 2015). About 25\% of low HDL was observed in men who were 60 and above, which was lower than aged 40-59 with low HDL of 30.2\% (Figure 6) (Carroll et al., 2015). The women in the age group of 20-39 had 11.7\% and 40-59 was 9.9\%, the 2 previous results were higher than women aged 60 and above with low HDL of 7.2\% (Figure 6) (Carroll et al., 2015).

The prevalence of elevated low HDL cholesterol was associated with increase in age and body mass index in children and adolescent (Nguyen et al., 2015). The prevalence of Low HDL cholesterol was found to be lower in women and girls than in men and boys.

\section{DISCUSSION}

Cardiovascular disease prevention should be initiated during early childhood to prevent the development of cardiovascular diseases later in life (Labarthe, 1999). It was estimated that $20 \%$ of children and adolescent (1 out of 5) had one of the abnormal cholesterol (high total cholesterol or low HDL cholesterol) (Nguyen et al., 2015). The low HDL cholesterol was the most abnormal among the cholesterol screening (Nguyen et al., 2015). In comparison, 19\% of adults had low HDL cholesterol (Carroll et al., 2015), which is higher than the children and adolescent (13.4\%) (Nguyen et al., 2015). In addition, it was estimated that $12 \%$ of adults had high total cholesterol (Carroll et al., 2015) while children and adolescents had 7.4\% (Nguyen et al., 2015). The adolescent aged 16 and 19 had high total cholesterol and low HDL cholesterol when compared with children aged 6-8 (Nguyen et al., 2015). It was observed from the review that obese children and adolescent had highest level of low HDL (5 times higher) as compared to children who are not obese (Nguyen et al., 2015). The prevalence of high total cholesterol was found to be lower in non-Hispanic black adults than non-Hispanic white and Hispanic (Carroll et al., 2015). Moreover, the prevalence of low HDL cholesterol was lower in non-Hispanic black men as compared to nonHispanic white and Hispanic men (Carroll et al., 2015). Furthermore, the prevalence of low HDL cholesterol was lower in non-Hispanic black and non-Hispanic Asian women when compared to non-Hispanic white and Hispanic women (Carroll et al., 2015). The prevalence of high total cholesterol was higher in non-Hispanic black, and nonHispanic Asian children as compared to non-Hispanic white and Hispanic children (Nguyen et al., 2015). The nonHispanic white and Hispanic children and adolescent had low HDL cholesterol which is higher the non-Hispanic black and non-Hispanic Asian (Nguyen et al., 2015). 
The best method to know the children and adolescent at risk of high cholesterol is through screening (Bloetzer, Bovet, Suris, Simeoni, Paradis and Chiolero, 2015). The intention of any screening exercise in a population that is healthy is to identify people at risk or who already had the disease but still in the early stage (Wald, 1999). Individuals who got screened and confirmed to have the disease will get intervention that will help prevent or treat the disease to stop future consequences (Wald, 1999). Early prevention of CVD during childhood help to identify children at risk of CVD through risky behaviors, and the development of early intervention program that will reduce the risk of CVD (Bloetzer et al., 2015).

The prevalence of abnormal cholesterol in children, adolescent and adults with different race and sex have been well documented but there is little information on the comparison of abnormal cholesterol among these groups. One of the factors used in monitoring the U.S health is the total cholesterol (Carroll et al., 2015) and according to one of the goals of Healthy people 2020 concerning cholesterol was to lower high total cholesterol by $13.5 \%$ (Klein, 2001). The goal was achieved in 2011-2014 when $12 \%$ of adults aged 20 and above had met the goal while adults aged 40-59 years (16\%) did not achieve this goal (Carroll et al., 2015). Furthermore, sex, race and Hispanic origin met the goal while the non-Hispanic white women (13.8\%) did not (Carroll et al., 2015). In addition, the risk factors for CVD in adults have been well documented but little evidence for CVD risk factors was shown in children and adolescent (Berenson, 1992). Elevated high total cholesterol and low HDL cholesterol are important risk factor for CVD (Bloetzer et al., 2015). The level of total cholesterol and HDL cholesterol are very important in clinical practice because of its importance in determining the health status of a patient (Carroll et al., 2015). Most physician measure cholesterol level and other factors like blood pressure, smoking history and weight to assess the risk of developing a CVD in both children and adult (Stone, 2014). The American academy of Pediatrics proposed that all children should be monitored for cholesterol (Expert Panel on Integrated Guidelines for Cardiovascular Health and Risk Reduction in Children and Adolescents, 2011). The continuous monitoring of abnormal cholesterol in children and adolescent will help the public health experts to make an informed decision on long term intervention in preventing CVD in adulthood and reduce the burden of health cost on the taxpayers (Nguyen et al., 2015).

\section{CONCLUSION}

The prevalence of low HDL cholesterol is higher in children, adolescent and adult male population as compared to the female participants whereas high total cholesterol was higher in the female as compared to the male which can be associated with high risk of cardiovascular diseases. The black adults, who are non-Hispanic had a lower prevalence of high total cholesterol than other race (Carroll et al., 2015). Furthermore, the black men, who are non-Hispanic in origin had low occurrence of low HDL cholesterol than others (Carroll et al., 2015). Prevalence of low HDL cholesterol was lower in women who are non-Hispanic black and Asian (Carroll et al., 2015). The non-Hispanic black and non-Hispanic Asian children and adolescent had a low prevalence of low HDL cholesterol than others while non-Hispanic white and Hispanic children and adolescent had low prevalence of high total cholesterol (Nguyen et al., 2015).

\section{ACKNOWLEDGEMENT}

I would like to express my sincere gratitude to Professor Mustafa Younis for his supervision throughout the course of this project and his encouragement to get it published.

\section{REFERENCES}

Assmann, G., Schulte, H., von Eckardstein, A. and Huang, Y. (1996). High-density lipoprotein cholesterol as a predictor of coronary heart disease risk: The PROCAM experience and pathophysiological implications for reverse cholesterol transport. Atherosclerosis, 124 Suppl., pp. S11-S20.

Berenson, G.S., Srinivasan, S.R., Bao, W., Newman 3 ${ }^{\text {rd }, ~ W . P ., ~ T r a c y, ~ R . E . ~ a n d ~ W a t t i g n e y, ~ W . A . ~(1998) . ~ A s s o c i a t i o n ~}$ between multiple cardiovascular risk factors and atherosclerosis in children and young adults. The Bogalusa heart study. New England Journal of Medicine, 338(23), pp. 1650-6.

Berenson, G.S., Wattigney, W.A., Tracy, R.E., Newman, W.P., Srinivasan, S.R., Webber, L.S., et al. (1992). Atherosclerosis of the aorta and coronary arteries and cardiovascular risk factors in persons aged 6 to 30 years and studied at necropsy (The Bogalusa Heart Study). American Journal of Cardiology, 70(9), pp. 851-8.

Bloetzer, C., Bovet, P., Suris, J.C., Simeoni, U., Paradis, G. and Chiolero, A. (2015). Screening for Cardiovascular Disease Risk Factors beginning in Childhood. BioMed Central, doi:10.1186/s40985-015-0011-2 
Carroll, M.D., Fryar, C.D. and Kit, B.K. (2015). Total and high-density lipoprotein cholesterol in adults, United States, 2011-2014. NCHS data brief, no 226. Hyattsville, MD, National Center for Health Statistics.

Chen, X., Wang, Y. (2008). Tracking of blood pressure from childhood to adulthood: a systematic review and meta-regression analysis. Circulation, 117(25), 3171-80.

Expert Panel on Integrated Guidelines for Cardiovascular Health and Risk Reduction in Children and Adolescents (2011). Summary report. Pediatrics, 128 Suppl 5, pp. S213-56.

Klein, R.J. and Schoenborn, C.A. (2001). Age adjustment using the 2000 projected U.S. population. Healthy People 2010 Statistical Notes, no 20. Hyattsville, MD, National Center for Health Statistics.

Labarthe, D.R. (1999). Prevention of cardiovascular risk factors in the first place. Prev Med., 29(6 Pt 2), pp. S72-8.

Li, S., Chen, W., Srinivasan, S.R., Bond, M.G., Tang, R., Urbina, E.M., et al. (2003). Childhood cardiovascular risk factors and carotid vascular changes in adulthood: the Bogalusa heart study. JAMA, 290(17), pp. $2271-6$.

McGill Jr, H.C., McMahan, C.A. and Gidding, S.S. (2008). Preventing heart disease in the 21st century: implications of the pathobiological determinants of atherosclerosis in youth (PDAY) study. Circulation, 117(9), pp. $1216-27$.

National Center for Health Statistics. National Health and Nutrition Examination Survey: NHANES $2011-2012$. Hyattsville, MD.

National Heart, Lung, and Blood Institute (NHLBI) (2012). Integrated guidelines for cardiovascular health and risk reduction in children and adolescents, ww.nhlbi.nih.gov/guidelines/cvd_ped/index.htm

Nguyen, D.T., Kit, B.K. and Carroll, M.D. (2015). Abnormal cholesterol among children and adolescents in the United States, 2011-2014. NCHS data brief, no 228. Hyattsville, MD, National Center for Health Statistics.

Stone, N.J., Robinson, J.G., Lichtenstein, A.H., Bairey Merz, C.N., Blum, C.B., Eckel, R.H., et al. (2014). ACC/AHA guideline on the treatment of blood cholesterol to reduce atherosclerotic cardiovascular risk in adults. A report of the American College of Cardiology/American Heart Association Task Force on Practice Guidelines. Journal American College of Cardiology, 63(25 Pt B), pp. 2889-934.

Wald, N.J., Hackshaw, A.K. and Frost, C.D. (1999). When can a risk factor be used as a worthwhile screening test? $B M J, 319$ (7224), pp. 1562-5.

WHO. Global Health Observatory (GHO): NCD mortality and morbidity. Available at: www.who.int/gho/ncd/mortality_morbidity/en. Accessed 16 Sept 2014. 